\title{
SOME POWER SERIES WITH RANDOM GAPS
}

\author{
PHILIP HOLGATE, ${ }^{*}$ Birkbeck College, London
}

\begin{abstract}
Power series $\sum z^{X_{n}}$ are studied, where $\left\{X_{n}\right\}$ is a strictly increasing integer-valued stochastic process.
\end{abstract}

RANDOM POWER SERIES; NATURAL BOUNDARY

The function theoretic properties of random functions are discussed in the monograph of Kahane (1985), and random power series in particular are surveyed by Lukacs (1975), Chapter 5. Walk (1968) drew attention to the parallels between probability 1 properties of random power series and those of certain deterministic lacunary series. Brief references to the properties of fixed lacunary series can be found in, for example Titchmarsh (1939), \$7.4. Arnold (1966) studied series in which the sequence of powers for which the coefficient is non-zero is specified deterministically, but the values of the coefficients are random. This note is about lacunary series of the form $f(z)=\sum_{0}^{\infty} z^{x_{n}}$ in which the gap lengths are random while the coefficients are all 1.

Corresponding to any random power series we define the mean function $\mu(z)=E f(z)$ and the real variance function $v(z)=E(f(z)-\mu(z))$, provided the relevant sums converge. The indices occurring in a realisation of $f(z)$ form a catalogue of the states visited by the sample sequence $\left\{X_{n}\right\}$ and $\mu(z)$ is a generating function for the expected numbers of visits of the process to the states.

Case 1. Suppose that $X_{0}=0, X_{n}=\sum_{j=0}^{n} Y_{j}$, and the $\left\{Y_{n}\right\}$ are independent, positive integer-valued random variables, with common p.g.f. $\pi(z)$. The corresponding mean series is $\mu(z)=\sum_{0}^{\infty} \pi^{n}(z)=1 /(1-\pi(z))$, which is singular at $z=1$.

Examples. For the uniform distribution on $1, \cdots, k, \mu(z)=k(1-z) /\{k-(k+1) z+$ $\left.z^{k+1}\right\}$. For the geometric distribution $p_{j}=(1-\theta) \theta^{j-1}, j \geqq 1$, we have $\mu(z)=(1-\theta z) /(1-z)$. The real variance is

$$
\begin{aligned}
\sum_{m=0}^{\infty} \sum_{n=0}^{\infty} E z^{X_{m}} \bar{z}^{X_{n}}-|\mu(z)|^{2} & =\sum_{m=0}^{\infty} E|z|^{2 X_{m}} \sum_{j=0}^{\infty}\left(z^{X_{j}}+\bar{z}^{X_{j}}\right)-\sum_{n=0}^{\infty}|z|^{2 X_{n}}-|\mu(z)|^{2} \\
& =\mu\left(|z|^{2}\right)\{\mu(z)+\mu(\bar{z})\}-\mu\left(|z|^{2}\right)-|\mu(x)|^{2} .
\end{aligned}
$$

With probability 1 the series diverges at $z=1$, and by Pringsheim's theorem, $f(z)$ has a singularity there. For any integer $k$ we can write

$$
f(z)=\sum_{j=0}^{k-1} z^{j} \Sigma^{(j)}\left(z^{k}\right)^{\left(X_{n}-j\right) / k}=\sum_{j=0}^{k-1} z^{j} f_{j}(z),
$$

where the summation $\Sigma^{(j)}$ extends over those $n$ for which $X_{n} \equiv j(\bmod k)$. Each $f_{j}(z)$ has the same probability distribution as $f\left(z^{k}\right)$, and hence with probability 1 has singularities at each

Received 7 April 1989; revision received 10 May 1989.

* Postal address: Department of Statistics, Birkbeck College, University of London, Malet St., London WC1E 7HX. 
$k$ th root of unity. The functions will only add up to cancel out the singularities if a fixed relationship holds between the $\left\{f_{j}(z)\right\}$. This is not so, since if $k-1$ of them are fixed, the $k$ th has positive conditional variance. Thus $f(z)$ has a natural boundary produced by the stochastic irregularity of the gap lengths. The examples show that a random series may have a natural boundary with probability 1 , but its mean series be analytically continuable. Let $\varphi(z)=f(z) / \mu(z)=(1-\pi(x)) f(z)$. Then $E \varphi(z)=1, \lim _{z \rightarrow 1} \operatorname{var} \varphi(z)=1$. Multiplying and dividing by $1-z$ we find $\lim _{z \rightarrow 1} \varphi(z)=\pi^{\prime}(1) \lim _{z \rightarrow 1}(1-z) f(z)$. Now $\pi^{\prime}(1)=E X_{1}$. Since $(1-z)$ can only cancel out a simple pole, $\varphi(z)$ has with probability 1 an essential singularity at $z=1$.

Case 2. Suppose now that $\left\{X_{n}\right\}$ is a stochastic branching process (Harris (1963)) whose offspring p.g.f. is $\pi(z)$, with expectation $m>1$. On taking expectations of both sides of the series for the mean, we find $\mu(z)=\sum_{n=0}^{\infty} \pi_{n}(z)$, where $\pi_{n}(z)$ the p.g.f. of $X_{n}$ is the $n$th functional iterate of $\pi(z)$. We can write this as a functional equation, $\mu(z)=z+\mu(\pi(z))$. Write $\mu(z)=\sum b_{j} z^{j}$ for $|z| \leqq r_{0}$. Suppose that $\pi(0)=0$, then $b_{0}=0$. Substitution into the functional equation gives the relation $b_{n}=\delta_{1 n}+\sum\left\{n ! b_{\sum k_{i}}\left(k_{1} ! \cdots k_{n} !\right)\right\}\left(p_{1} 1 !\right)^{k_{1}} \cdots\left(p_{n} n !\right)^{k_{n}}$ (summation over all positive integral solutions of $k_{1}+2 k_{2}+\cdots+n k_{n}=n$ ), $=\delta_{1 n}+$ $A_{n}\left(b ; p_{1} \cdots p_{n}\right)$, where $\delta_{1 n}$ is a Kronecker symbol and $A_{n}$ is the $n$th Bell polynomial (Riordan (1958), p. 34). The first few coefficients are found to be: $b_{1}=1 /\left(1-p_{1}\right), b_{2}=p_{2} /\left(\left(1-p_{1}\right)(1-\right.$ $\left.\left.p_{1}^{2}\right)\right), b_{3}=p_{3} /\left(\left(1-p_{1}\right)\left(1-p_{1}^{3}\right)\right)+2 p_{1} p_{2}^{2} /\left(\left(1-p_{1}\right)\left(1-p_{1}^{2}\right)\left(1-p_{1}^{3}\right)\right)$. The mean series cannot be finite at $z=1$, for that would imply $\mu(1)=1+\mu(1)$. An expression can be derived for $v(z)$ in this case, but it is less simple than in Case 1.

It follows from the strong law of large numbers that $X_{n+1} / X_{n} \rightarrow m$ with probability 1 , and therefore the sequence has Hadamard gaps and a natural boundary. However, it is still possible for the mean series to be analytically continuable.

Examples. For the zero-modified geometric distribution it is possible to obtain an explicit expression for $\pi_{n}(z)$ for all $n$, namely

$$
\pi_{n}(z)=1-m^{n}(1-q)\left(m^{n}-q\right)^{-1}\left\{1-(1-q) z\left(m^{n}-q-\left(m^{n}-1\right) z\right)^{-1}\right\}
$$

where

$$
q=\pi(0), \quad m=\pi^{\prime}(1) .
$$

It can be seen that in this case $\mu(z)$ has poles at $z=\left(m^{n}-q\right)\left(m^{n}-1\right)^{-1}$ for $n=1,2, \cdots$, and hence that $z=1$ is a limit point of poles. However, $\mu(z)$ is regular everywhere else on its circle of convergence. For the Petersburg distribution, $\operatorname{Pr}\left(X=2^{n}\right)=\left(\frac{1}{2}\right)^{n+1}, n=0,1, \cdots$, the p.g.f. $\pi(z)=\sum\left(\frac{1}{2}\right)^{n+1} z^{2^{n}}$ has a natural boundary at $|z|=1$. Then $\pi(z)$ becomes unbounded as $z \rightarrow z_{0}$ for any $z_{0}$ on the unit circle. Thus $\mu(z)$ is unbounded as $z \rightarrow z_{0}$, and has a natural boundary.

The similarity in behaviour of random power series and deterministic lacunary series investigated here reflects a wider parallelism between the latter and general sequences of independent or nearly independent random variables. This is seen most strongly in the probability theoretic and function theoretic formulations of the law of the iterated logarithm. It is discussed in the references cited by Bingham ((1986), Section 20.1), in particular in the papers of Salem and Zygmund (1950), and Makarov (1985).

\section{References}

ARNOLD, L. (1966) Konvergenzproblemen bei zufälligen Potenzreihen mit Lücken. Math. Zeitschr. 92, 356-365.

Bingham, N. H. (1986) Variants of the law of the iterated logarithm. Bull. London Math. Soc. 18, 433-467.

HARris, T. E. (1963) Branching Processes. Springer-Verlag, Berlin.

Kahane, J.-P. (1985) Some Random Series of Functions, 2nd edn. Cambridge University Press.

Lukacs, E. (1975) Stochastic Convergence, 2nd edn. Academic Press, New York. 
MaKarov, N. G. (1985) On the distortion of boundary sets under conformal mapping. Proc. London Math. Soc. (3) 51, 368-384.

RioRdan, J. (1958) Combinatorial Analysis. Wiley, New York.

SALEM, R. AND ZYGMUND, A. (1950) La loi du logarithme itéré pour les séries trigonométriques lacunaires. Bull Sci. Math. 74, 209-224.

Trtchmarsh, E. C. (1939) The Theory of Functions, 2nd edn. Oxford University Press.

WALK, H. (1968) Uber das Randverhalten zufälligen Potenzreihen. J. reine angew. Math. 230, 66-103. 